\title{
Wireless Allowing Data and Power Transfer
}

C. Dehos ${ }^{\star a}$, E. Locci ${ }^{b}$, R. Brennerc, D. Dancilac, P. De Lurgiod, Z. Djurcic ${ }^{d}$, G. Drake ${ }^{d}$, J.L. Gonzalez Gimenez ${ }^{\text {b }}$ L. Gustafsson ${ }^{c}$, D.W. Kim ${ }^{\text {e, U. Pfeiffer }}{ }^{f}$, D. Röhrich ${ }^{g}$, D. Rydberg $^{c}$, A. Schöning ${ }^{\text {h }}$, A. Siligaris ${ }^{a}$, H.K. Soltveit ${ }^{\text {h }}$, K. Ullaland ${ }^{\mathrm{g}}$, P. Vincent ${ }^{\mathrm{a}}$, P.R.

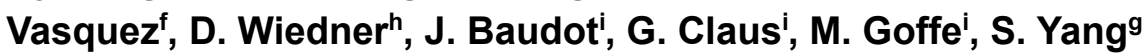

a Université Grenoble-Alpes, CEA-Leti, Minatec Campus, Grenoble, France; ${ }^{b}$ CEA/DRF/IRFU/SPP, Gif-sur-Yvette, France; ${ }^{c}$ Uppsala University, Sweden; ${ }^{d}$ Argonne National Laboratory, Argonne, IL 60439, USA ; ${ }^{e}$ Gangneung National University, Korea; ${ }^{f}$ University of Wuppertal, Germany, ${ }^{g}$ University of Bergen, Norway; ${ }^{h}$ University of Heidelberg, Germany, ${ }^{\mathbf{i}}$ Institut Pluridisciplinaire Hubert Curien, Strasbourg, France

E-mail: cedric.dehosecea.fr

The WADAPT consortium (Wireless Allowing Data and Power Transfer) was created to study wireless (multi-gigabit) data transfer for high-energy physics applications (LoI, CERN-LHCC-2017-002; LHCC-I-028.-2017). Emerging millimetre wave technologies allow fast signal transfer and efficient partitioning of detectors in topological regions of interest. Large bandwidths are available at those frequencies, allowing very high data rates at short range and conveniently substituting a mass of materials (cables and connectors). The Wadapt initiative aims at building proof of concept for use in future HEP experiments. For vertex detectors at HL-LHC, the bandwidth of $60 \mathrm{GHz}$ is adequate and commercial products are already available, providing 6 Gbps data links. Products have been tested for signal confinement, crosstalk, electromagnetic immunity and resistance to radiation. An HEP dedicated $60 \mathrm{GHz}$ Integrated Chip is being built in Heidelberg, using $130 \mathrm{~nm}$ SiGe BICMOS technology. It should assess the feasibility and performance of the wireless link and establish solid foundation for designing the final reading system. At longer terms, $140 \mathrm{GHz}$ bands could also be used for higher data rates (>100 Gbps) for future FCC applications. Wireless reading could widespread to many detectors, with the possibility of adding intelligence on the detector to perform fourdimensional reconstruction of the traces and vertexes online, in order to attach the traces to their vertex with great efficiency even in difficult experimental conditions. The WADAPT project includes also a long-term step aimed at transmitting energy wirelessly. This would create a new paradigm for the transmission of data and power in particle physics detectors.

40th International Conference on High Energy physics - ICHEP2020

July 28 - August 6, 2020

Prague, Czech Republic (virtual meeting) 


\section{Introduction}

Future High Energy Physics (HEP) experiments shall tackle unprecedented event rates and precision, raising the challenge of the huge data readout from detectors. E.g., the data rate needed to readout all 1-2 hit clusters in the upgraded ATLAS silicon micro-strip tracker is expected between $50-100 \mathrm{~Tb} / \mathrm{s}$ with more than 10000 data links at $5 \mathrm{Gbps}$, in a small volume of $1 \mathrm{~m}$ radius pixel detector. Thus the power and data cabling becomes hardly manageable, increasing the material budget, creating multiple scattering, nuclear interactions and dead-zone areas. Besides the data aggregation and routing in the axial direction of the collider generates unwanted delays. In this context, we proposed in the framework of the WADAPT initiative to replace wired and optical interconnections by high-speed short-range wireless connectivity. The recent advances in semiconductor technologies, as well as in antennas and packaging, allows now Radio Frequency systems to operate at higher frequencies in the millimeter wave $(\mathrm{mmw})$, providing unprecedented bandwidth and data rates at short range. The relevance of the $\mathrm{mmw}$ wireless readout will be argued hereafter, through proposition of use case and integration scheme, and some preliminary tests and measurements.

\section{Millimeter Wave Wireless technology}

According to the Shannon-Hartley theorem, the capacity (data rate) of a wireless link is limited by the channel bandwidth and signal to noise ratio (SNR). For many years the trend was to get closer to this capcity limit by increasing the order of modulation at short/medium range (high SNR). Besides the succesive 802.11 working groups proposed many realeases in ISM bands at 2.4 and $5.6 \mathrm{GHz}$ with the purpose of increasing the data rates, based on more complex modulations.

The recent advances in low cost, mass market CMOS technologies allows now to design RFIC chips that operates higher in frequencies, in the mmw band (30-300 GHz). Below $90 \mathrm{~nm}$ width, the transistor cut-off and maximum frequencies (Ft/Fmax) of the CMOS technologies allows active designing up to $\sim 70 \mathrm{GHz}$ and up to $\sim 180 \mathrm{GHz}$ below $45 \mathrm{~nm}$. Technologies below $22 \mathrm{~nm}$ may suffer from thiner back-end limitation, degrading the passive components performances.

The gain bandwidth product is also limited by the technology. As a rule of thumb, a relative bandwidth of 10 to $20 \%$ can be assumed for CMOS design. Below $6 \mathrm{GHz}$, frequency bands are ususally limited to 20 or $40 \mathrm{MHz}$ for $\mathrm{WiFi}$, with a maximum of $160 \mathrm{MHz}$ for the $802.11 \mathrm{ac}$ standard. The use of adanced modulation scheme and multiple data streams (MIMO) requires intensive signal processing, increasing the complexity of the chipset design, the size as well as the power consumption. At millimeter wave the frequency bands cover multiple $\mathrm{GHz}$ as illustruted on figure 1. In the USA the FCC regulation has extended the $60 \mathrm{GHz}$ band to $57-71$ $\mathrm{GHz}$ for unlicensed operation. These huge bandwidths allow huge data capacity while keeping low order of modulation with good power efficiency.

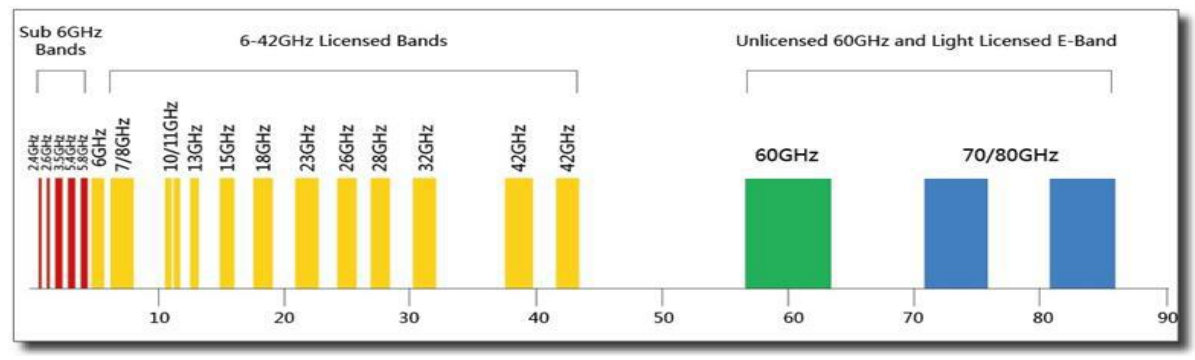

Figure 1: available frequency bands between 2 and $100 \mathrm{GHz}$ 
Another advantage of going higher in frequency is to profit from the small wave length. The antenna size is typically linked with half the wavelength. At $60 \mathrm{GHz}$, compact antenna of $3 \mathrm{~mm}$ can be integrated in silicon (System on Chip), or with the chip in the package (System in Package).

Figura 2: Silicon interposer with integrated antennas [1] (silicon chip on top)

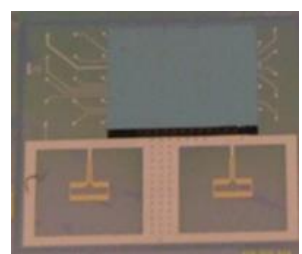

Two main transceiver architectures may be considered at mmw frequencies. Coherent architecture requires precise frequency synthesis (using Phase Locked Loop) and time and frequency synchronization mecanism achieved by base band crcuit. This architecture allows complex amplitude and phase modulations, achieving high spectral efficiency, but require high power consumption. On the contrary non coherent architecture allows amplitude modulation only (On Off Keying, Amplitude Shift Keying or Pulse Amplitude Modulation). The receiver can implement a simple envelope detector, a compartor and digital interface, without any frequency reference or digital base band processing. The quadratic envelope detector provides negative gain, so that the overal receiver noise figure increases and affects the receiver sensitivity. For this reason this architecture is more dedicated to short range and low power communication.

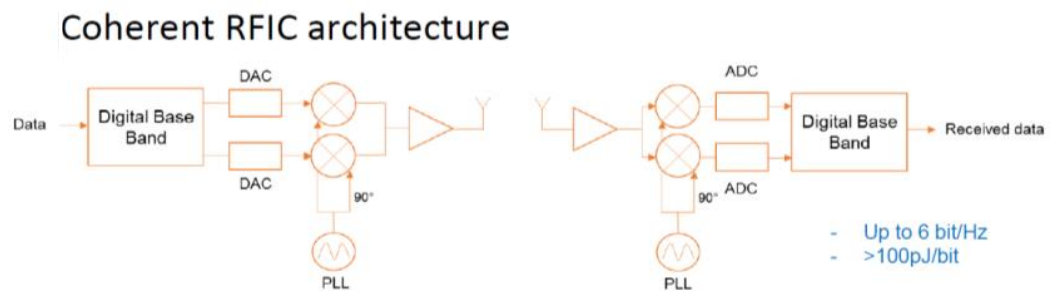

Non coherent RFIC architecture (On/Off keying)

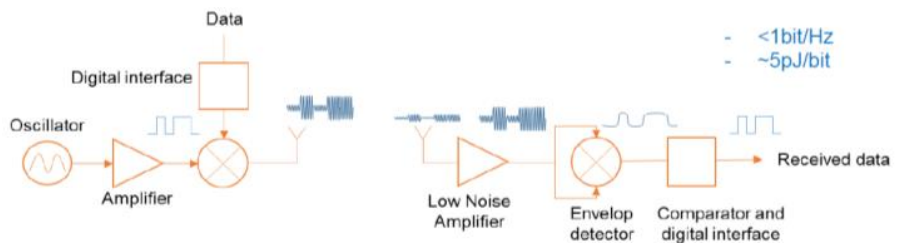

Figure 3: coherent and non-coherent $R F$ architecture schematics

In the last decade there has been tremendous advances in silicon technologies that have made it possible to build high performance RF transceivers operating in the millimetre-wave band. Non coherent architecture brings high data rate (up to $10 \mathrm{Gps}$ at $60 \mathrm{GHz}$ ) and low power $(<10 \mathrm{pJ} / \mathrm{bit}$ ) for short range applications. Recent advance in antenna and packaging design alows the integration of the mmw antenna on chip ([2]) or in package ([1]) with good efficiency. Commercial products are also now available for mass market applications with 3 main competitors (SiBeam, Keyssa and STMicroelectronics), proposing different integration schemes function of the application. Strong efforts have also been put on the compatibility with high/low speed digital protocols (USB3, PCIe, Gig Ethernet, UART) for a seamless cable replacement.

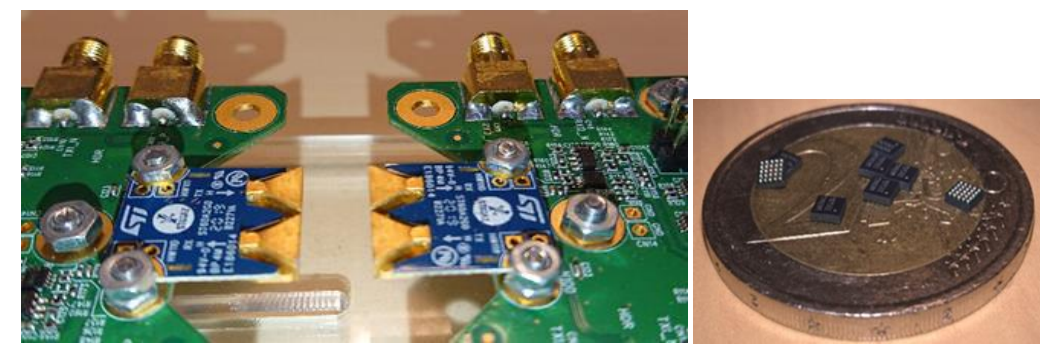

Figure 4: ST Microelecronics ST60 contacless connectivity transceiver in BGA (right) 
The above mentioned commercial solution does not guarantee long time reliability of operting in harsh environment, as the one of silicon trackers. A $60 \mathrm{GHz}$ wireless transceiver dedicated to HEP is in its final step of development at Heidelberg University ([3]). The transceiver designed with IBM $130 \mathrm{~nm}$ SiGe HBT BiCMOS technology shall achieve multi Gbps radial data transmission inside the ATLAS silicon strip detector, making a first level track trigger feasible. Both technology and RF architecture choices should brings more reliability and robustness to irradiations. The first prototype would be able to handle a data-rate of about 3.5 Gbps over a link distance of $1 \mathrm{~m}$.

Research is progresssing ([4]) towards very high data rate wireless communication systems, using channel aggragation or channel bonding in W or D-band (110-170 GHz), where huge bandwidths (32 $\mathrm{GHz}$ aggregated) are available for short range communications. Channel bonding is particularly relevent for the aggregation of data from multiple detectors. 16 channels of 7 Gbps can be aggragated in D-band transceiver. The data can be sent over a few tens of meter range using directive planar antennas, such as transmit arrays ([5]), or over light plastic waveguide material ([6]).

\section{Wireless readout}

The HEP present and future experiments requires more and more bandwidths. It is especially true for highly granular tracking detectors operated at high beam luminosities. As an example, the ATLAS Phase II New Inner Tracker Pixel Detector is expected to gather in 1m radius five-barrel layers, four end-cap rings and a silicon strip detector with four layers and six end-cap disks. The readout should operate at up to $4 \mathrm{MHz}(25 \mu \mathrm{s}) \mathrm{L} 0$ rate. At downlink a broadcast trigger and control signal will be sent at $160 \mathrm{Mbps}$ while 10k up-links are expected at $5 \mathrm{Gbps}$. The data readout problematic is investigated in [7], based on wired transmission strategy. Different cabling schemes are evaluated with the objectives of $5 \mathrm{Gbps}$ data transmission per cable, while reducing as much as possible the materials within the small detector volume. As an alternative [8] proposed to replace the innermost dense wired network by a multi-hop point-to-point wireless network, streaming the data radially from the center to outer enclosure.
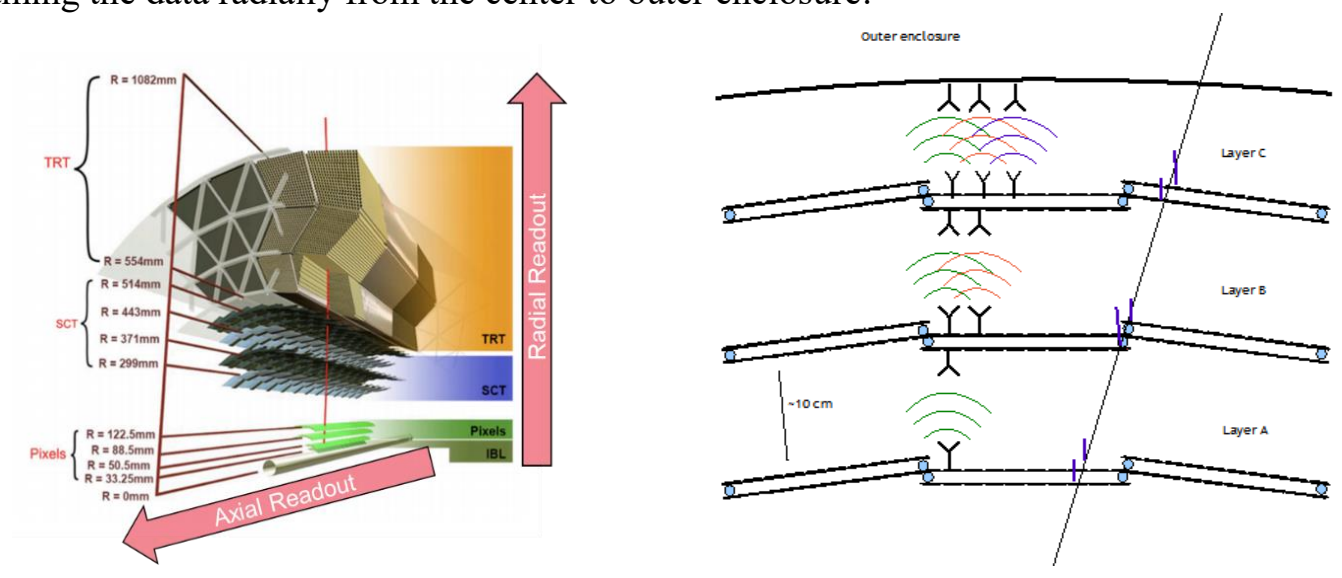

Figure 5: proposed wireless readout network schemtic from [8]

The short links are in the order of $10 \mathrm{~cm}$ range, allowing the use of low power non-coherent transceiver chips from chapter 2 with compact antennas. Signal is routed through each layer from the bottom receiver to the transmitter at top side. This multi-hop concept requires that the wave front stay confined between the two considered layers, and does not cross talks with neighbors.

Feasibility studies regarding the integration of the $60 \mathrm{GHz}$ wireless technologies in silicon tracking detector was performed in [9], based on a commercial coherent transceiver from Hittite. Several aspects relevant for the implementation of $60 \mathrm{GHz}$ links were studied: transmission losses, interference effects, absorbing materials and the influence of the antenna design and directivity. Error free transmission can be obtained with multiple transceivers spaced by $5 \mathrm{~cm}$ pitch using directive antenna scheme and absorbing materials. More recently, tests has been conducted using 
non-coherent transceiver prototypes from STMicroelectronics, with the aim of: reducing the power consumption, reducing the interconnection latencies, ease the digital interfacing with current detectors. The performances of non-coherent chips was measured at 1-5 cm range using low directivity antenna. The transmitter was fed by a SLVS $8 \mathrm{~b} / 10 \mathrm{~b}$ encoded signal at $5 \mathrm{Gbps}$. At the receiver output the binary signal exhibits less than 75 ps total jitter, a less than 35 p s $20-80 \%$ rising/falling time and less than $1 \mathrm{~ns}$ latency (without the cables). $10 \mathrm{~cm}$ range can be obtained using $10 \mathrm{~dB}$ gain antenna.
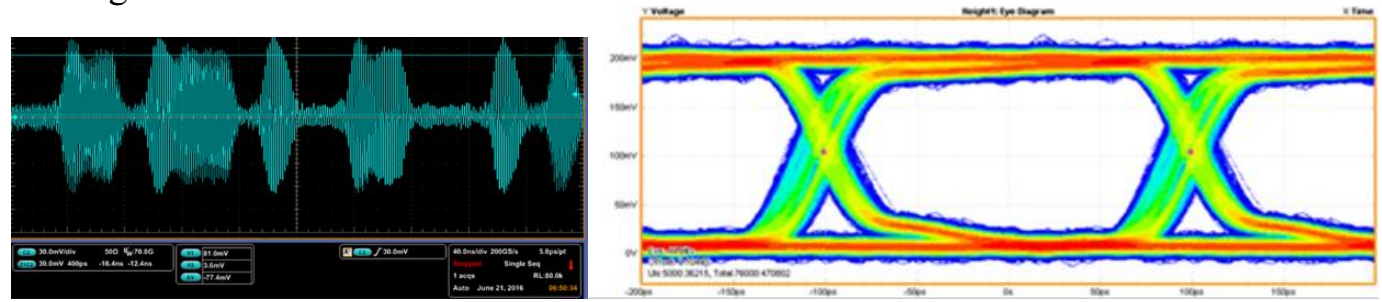

Figure 6: emitted waveform (top) and received eye diagramm (bottom) for 5Gbps wireless link

Non-coherent transceiver was then interfaced with Minimum Ionizing MOnolithic Active pixel Sensors (MIMOSA) from IPHC Strasbourg [10], that use the epitaxial layer of standard CMOS (TowerJazz High Resistivity $180 \mathrm{~nm}$ technology) processes as detecting sensitive volume. The pixel detector board provides $5 \mu$ s refreshment rate and generates up to 3 Gbps data rate. Signal integrity tests were carried out with detector feeding a wireless link. No error was reported at oscilloscope. Different materials were then introduced between the antennas. Signals well passed through the thin flex Kapton PCB that embeds chips, but not through low-density carbon foam that bears the detector. These results validates the proposed readout architecture, whose principle is to isolate every detector layer and export data through multiple hops between layers. At longer terms, RF transceiver may be integrated within the detector chip. Antenna could be also part of the chip with good efficiency, assuming the high resistivity substrate of the CMOS backend material. Pixel detectors still need to receive clock or trigger reference from the network. It could be worth then modifying the $60 \mathrm{GHz}$ transceiver, so that the clock is also transmitted wirelessly from chip to chip on the carrier by harmonic injection locking.
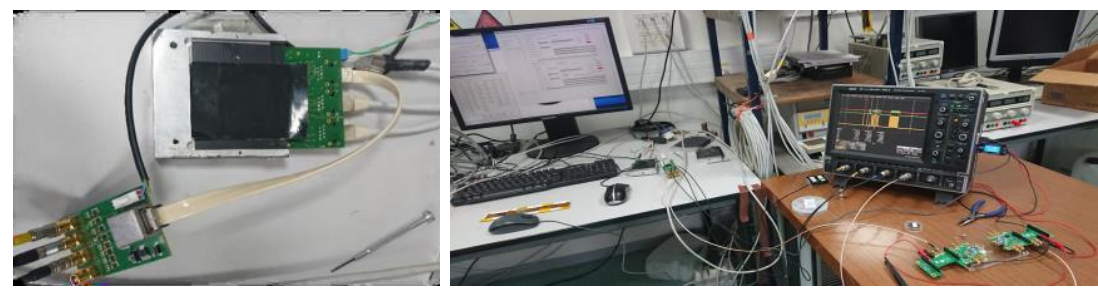

Figure 7: wireless data transmission interfacing with MIMOSA detector

Proton irradiation tests were conducted at Turku Cyclotron on CMOS 65nm commercial chip from STMicroelectronics with encouraging results [10]. The chip received a simulated energy dose of $19 \mathrm{Mrad}$ (target fluence of $1 \mathrm{e} 14 \mathrm{proton} / \mathrm{cm}^{2}$ ). Transmission performance was measured before, during and after irradiation. If some transmission errors was observed during the irradiation, the chip recovered error free transmission after irradiation with small gain degradation.
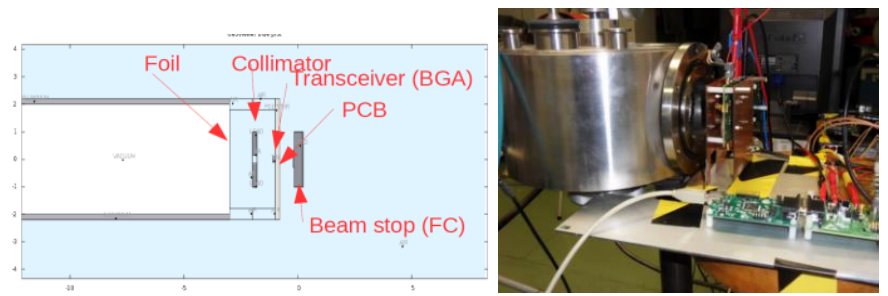

Figure 8: irradiation tests at Turku Cyclotron 


\section{Conclusions}

The recent advances in millimeter wave technologies allows low latencies, high data rate and low power wireless data transmission at short range. The association of non-coherent RF architecture and directive antennas are particularly relevant for the considered innermost part of the readout network, based on multi-hop transmission. Early feasibility tests with prototypes and commercial products show no deadlock to the use of these wireless technologies in HEP experiments, paving the way to early deployments and proof of concept using hardened RFICs and associated electronics. The mmw technological roadmap shows skyrocketing data rates using higher frequency bands above $100 \mathrm{GHz}$, challenging optical links for data aggregation and routing at short to medium range.

Technologies should be mature enough to be envisaged as a part of the readout network of next big HEP experiments, providing flexibility and fast triggering capabilities.

\section{References}

[1] L. Dussopt et al., "Silicon interposer with integrated antenna array for millimeter-wave short-range communications," 2012 IEEE/MTT-S International Microwave Symposium Digest, Montreal, QC, 2012, pp. 1-3, doi: 10.1109/MWSYM.2012.6259424.

[2] A. Siligaris et al., "A $60 \mathrm{GHz}$ UWB impulse radio transmitter with integrated antenna in CMOS65nm SOI technology," 2011 IEEE 11th Topical Meeting on Silicon Monolithic Integrated Circuits in RF Systems, Phoenix, AZ, 2011, pp. 153-156, doi: 10.1109/SIRF.2011.5719303.

[3] H. K. Soltveit et.al., "Towards Multi-Gigabit readout for the ATLAS silicon microstrip detector", Nuclear Science Symposium and Medical Imaging Conference (NSS/MIC), 2013 IEEE.

[4] Gonzalez-Jimenez et. al. "Channel-bonding CMOS transceiver for $100 \mathrm{Gbps}$ wireless point-to-point links" EURASIP Journal on Wireless Communications and Networking

[5] F. Manzillo, et. al. "Low-cost, High-Gain Antenna Module Integrating a CMOS Frequency Multiplier Driver for Communications at D-band," 2019 IEEE Radio Frequency Integrated Circuits Symposium (RFIC), Boston, MA, USA, 2019, pp. 19-22, doi: 10.1109/RFIC.2019.8701772.

[6] F. Voineau et al., "A $12 \mathrm{~Gb} / \mathrm{s}$ 64QAM and OFDM compatible millimeter-wave communication link using a novel plastic waveguide design," 2018 IEEE Radio and Wireless Symposium (RWS), Anaheim, CA, 2018, pp. 250-252, doi: 10.1109/RWS.2018.8305001.

[7] Francisca Muñoz Sánchez, "Module and electronics developments for the ATLAS ITk pixel system", 19th iWoRiD, 3rd July 2017, Krakow, Poland

[8] "Multigigabit wireless transfer of trigger data through millimetre wave technology" R. Brenner and S Cheng 2010 JINST 5 c07002

[9] H. Pernegger, "Monolithic pixel development in TowerJazz $180 \mathrm{~nm}$ CMOS for the outer pixel layers in the ATLAS experiment", Nuclear Instruments and Methods in Physics Research Section A: Accelerators, Spectrometers, Detectors and Associated Equipment, Volume 92

[10] S. Dittmeier et al., "Feasibility studies for a wireless $60 \mathrm{GHz}$ tracking detector readout", Nucl.Instrum.Meth. A830 (2016) 417-426 